\title{
Spatial Distribution Patterns of Genes in Intracardiac Neurons
}

\author{
Rohan Patil \\ Charter School of Wilmington, 100 N Dupont Rd, Wilmington, DE, 19807, USA ; 17rspatil@gmail.com
}

ABSTRACT: Cardiovascular disease is the number one cause of deaths in the United States. Now, neural circuits are being studied to understand their functions in relation to cardiovascular diseases. Intracardiac neurons (ICN) are part of a circuit that acts as an intermediary between the vagus nerve and cardiac function. Specific ion channels have been previously identified as vital to electrical conductivity in many areas throughout the body. Assuming these ion channels exhibit similar function in the heart, the ICN can be studied for the expression of genes that encode for functions relevant to electrical activity. This study explored that if levels of gene expression for specific ion channels is assessed with respect to location in different samples of cells from a rat ICN, then a connection can be identified between the location of the specific gene expression and function. By statistically analyzing gene expression profiles from many single neurons in the rat ICN, two families of genes were identified that exhibited unique properties concerning the sinoatrial node and were functionally related to electrical activity within the body. Subsequent research will be needed to understand how an alteration in this circuit can cause differences in cardiac function.

KEYWORDS: Cellular and Molecular Biology; Neurobiology; Intracardiac Neurons; Ion channels in Intracardiac Neurons.

\section{Introduction}

Cardiovascular disease, be it coronary heart disease or heart attacks, is the number one cause of deaths in the United States. One person dies every 37 seconds from cardiovascular disease. ${ }^{1}$ This is why many organizations such as the NIH have invested hundreds of millions of dollars into research on cardiovascular components such as studies related to the vagus nerve which is functionally responsible for cardiac function. Currently, neural circuits are being studied to understand their functions in relation to cardiovascular diseases. In fact, Andrew Armour, MD, $\mathrm{PhD}$, has published a report showing that neuronal circuits play a role in controlling cardiac function. ${ }^{2}$ Indeed, the research initiative, SPARC (Stimulating Peripheral Activity to Relieve Conditions) manipulates electrical activity in nerves for better cardiac function. Our study, which analyzes a neuronal circuit in the heart, falls under this project. Intracardiac neurons are part of a circuit that acts as an intermediary between the vagus nerve and cardiac function. ${ }^{2}$ Cardiac neurons are organized into ganglia, called ganglionated plexi (GPs), where a network of 200-1000 neurons form an integration center and innervate the sinoatrial and atrioventricular nodes. ${ }^{1}$ Functionally similar but anatomically variant, each neuron in GPs expresses genes that regulate the number of ion channels present in the $\mathrm{ICN}{ }^{3}$ Once DNA is transcribed in the nucleus, mRNA translation occurs in the endoplasmic reticulum and the golgi apparatus transports the ion channels to the cell's membrane where they interact with other proteins such as anchoring elements or kinases to morph into a functional channel. ${ }^{4}$ Ion channels are proteins that allow for the exchange of chemicals across the membrane of the cell to produce an action potential, which is an electric signal that triggers the compression of the heart. ${ }^{5}$ The ion channels in neurons likely contribute to the heart rate in humans. Specifically, the sinoatrial node and myocardium consist of voltage-gated sodium, calcium, and potassium channels which contribute to neurotransmitter docking, vesicle release, and repolarization and hyperpolarization after an action potential. ${ }^{6}$ Neurons in the ICN are the source of the series of depolarizations which make up the action potentials powering blood vessel contractions in the sinoatrial node and in turn, contractions of the heart. 3D neuroanatomical information showing the ICN in relation to other anatomical features is necessary to understand the functional connectivity of the neurons and to elucidate their functional circuit organization. ${ }^{7}$ In this study, the gene expression of ion channels in intracardiac neurons in a rat heart were analyzed to support the potential relation of gene expression to location in the heart. By analyzing what genes are expressed more than others, a connection between gene expression and spatial distribution can be identified and through this, the ICN can become a target for treatments of various cardiac diseases generated by irregularities in electrical activity.

\section{Methods}

To begin, a model of the rat's heart was created to visualize the location of the ICN in relation to the various anatomical structures within the heart. To create a 3D model of the rat heart, a Knife Edge Scanning Microscope (KESM) was used to image sections of the heart, giving us 2D images (Figure 1). By stacking these images into TissueMapper, a custom software, the images were modified to create a $3 \mathrm{D}$ visualization of the rat heart. To modify the image stack, an outline was formed around all visible structures and the outline of the heart for each individual image acquired (853 in our rat heart). Next, the contours were aligned into a stack to create a $3 \mathrm{D}$ visualization of the rat heart (Figure 2). To do this, the aorta and the left ventricle were used to align the contours because the aorta was consistently visible throughout most of the image stack. However, when the aorta faded from view, the left ventricle appeared consistently and was therefore used. After the con- 
touring and alignment was complete, a 3D structure of a rat's heart with the sampled neurons displayed in spatial relation to the anatomical structures of the heart was visible (Figure 3).

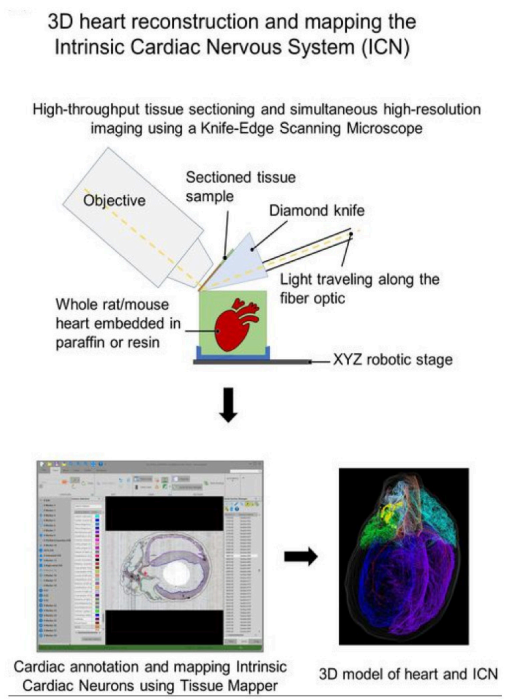

Figure 1: KESM described visually in the process of $2 \mathrm{D}$ imaging to later be compiled into a precise 3D model through TissueMapper.

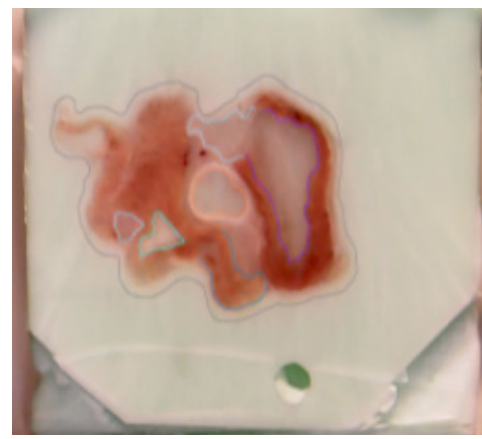

Figure 2: An image from the stack that has contours drawn outlining the interior structures and the outline of the heart.
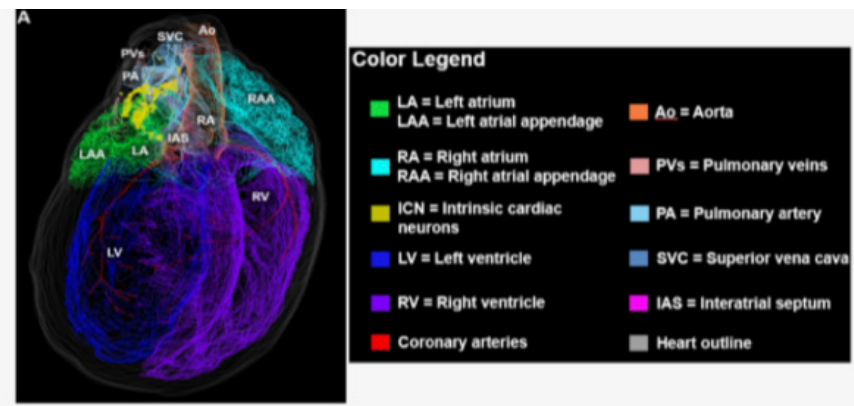

Figure 3: The 3D model of the rat heart with the ICN shown in relation to other anatomical structures. It innervates the $\mathrm{SA}$ and $\mathrm{AV}$ node, indicating a strong influence on the electrical conductivity of the heart.

To analyze the expression of genes, the raw data acquired from transcribing the RNA through RT-QPCR was normalized by finding the median among the values of gene expression and standardizing the values of 40 ion channel genes under study from various other preliminary projects compared to the median. Once normalized, the data was compiled into a '.csv' file to be readable in the $\mathrm{R}$ programming language. We used RStudio to run "R" and XQuartz to visualize the gene expres sion map. Using the gene name as an input, the code produces an image of the sampled neurons colored with different shades of red and blue, showing the expression of the specific gene.

\section{Results and Discussion}

The expression data of ion channels in the ICN of a rat heart were analyzed. The experiment tested whether the gene expression of ion channels demonstrated a spatial localization specific to certain ganglia. The following images show the gene expression profile for the HCN and CACNA gene families, which play a pivotal role in electrical conductivity. Each gene is shown as a whole in a XYZ coordinate plane and an arrow points to a cluster estimated to be the sinoatrial node area.

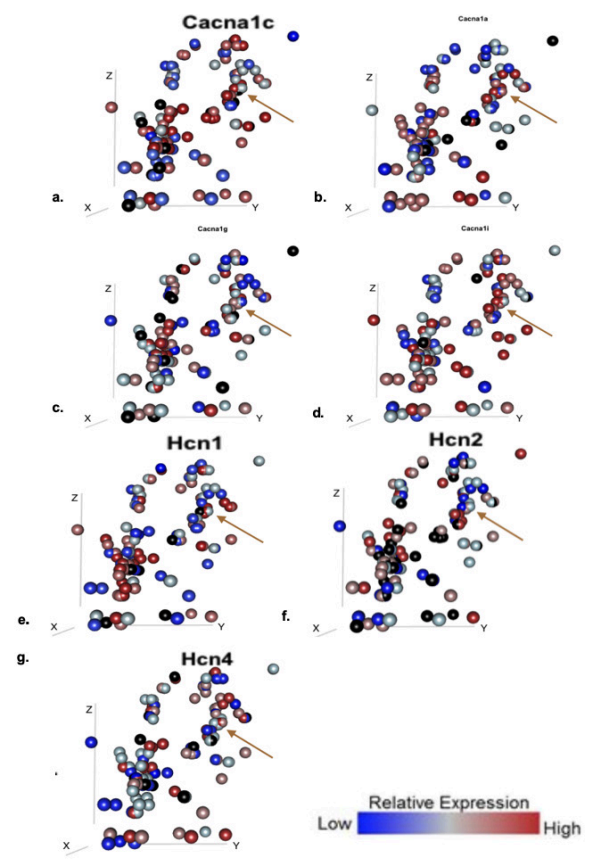

Figure 4: Gene expression maps of (a)Cacna1a (b)Cacna1c (c)Cacna1g (d) Cacna1i (e)Hcn1 (f)Hcn2 (g)Hcn4. Individual spheres represent individual sampled neurons. (h)Relative gene expression key; Extreme red signifies very high gene expression and extreme blue signifies very low gene expression. Lighter shades signify a less extreme of their respective color. Gene expression was consistently high across all selected genes in these clusters, demonstrating the select 7 genes playing a major role in regulating electrical conductivity in the heart.

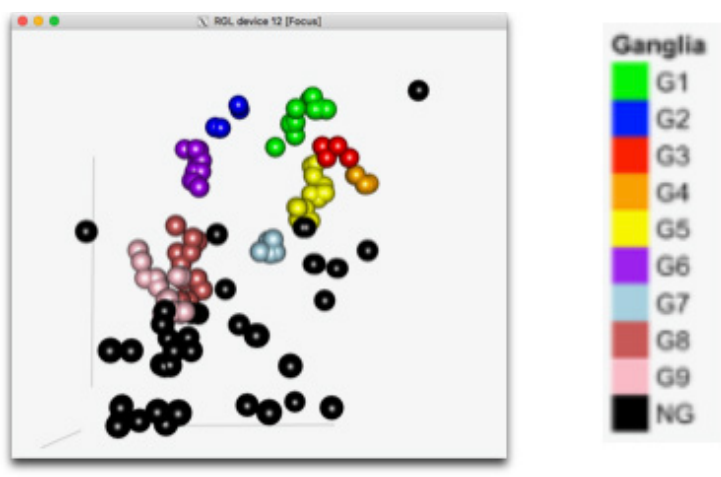

Figure 5: The separation of all 151 sampled neurons into 9 different color-coded ganglia. 3 ganglia making up the SA node cluster indicate the communicative effort of more than one ganglion to control electrical conductivity in the heart. 
The neuronal circuit in the heart was analyzed specifically for certain genes encoding ion channels and receptors. While looking at the 3D images of the genes for ion channels (Figure 4), there were a handful of genes that were expressed highly around the sinoatrial node, as pointed out by arrows. The sinoatrial node is likely located in the upper right corner of the 3D images (G3, G4, G5 in Figure 5) and it is also referred to as the heart's natural pacemaker. This is because it functions as the main natural electrical stimulator of the heart, causing the heart to contract and thus keep blood oxygenated. ${ }^{8}$ The analyzed genes include Hcn1, Hcn2, Hcn4, Cacna1a, Cacna1c, Cacna1g, Cacna1i. These particular genes code for ion channels that have a major role in sending and transmitting electrical signals in the heart. Research shows that $\mathrm{HCN}$ encoded ion channels play a critical role in the generation and conduction of the electrical impulse and the physio pathological process of some cardiac diseases such as heart failure and atrial/ventricular fibrillation. ${ }^{9}$ The data appears to show some sort of spatial distribution observed in the genes for ion channels around the sino-atrial node, specifically $\mathrm{HCN}$ genes as there was a more noticeable pattern in these than CACNA genes, visually. Analyzing the images above, it could be concluded that there is a clear visual pattern in high (extreme red) gene expression in the top right corner of the images. Each gene is significantly expressed in a small region of the cluster (Figure 4).

This tells us that there is an opportunity to further investigate additional genes because there seems to be some sort of spatial pattern in this particular case. This study can further our understanding of the function of ion channels in the heart. We have extrapolated this spatial analysis across several rat and pig hearts, furthering our basis for a consistency in these specific ion channels controlling the sinoatrial node. The constant observation of similar expression in genes across multiple species and hearts eliminates several sources of errors in RNA expression when obtaining measurements. Our recordings of neurons in GPs innervating the SA node, as discovered through $\mathrm{Z}$-axis slicing and microscope comparisons, differ significantly from those of other anatomical features, indicating a specialization for ion channels present in creating and containing action potentials. While precise synaptic communications cannot be measured, neurotransmitter receptors have demonstrated an imbalance in ICN, providing an opportunity for further analysis. Expanding our analysis to more genes for ion channels can also help determine additional patterns. In the future, we would like to visualize the gene expression of ion channels and other genes and compare the patterns between a healthy rat heart and a diseased rat heart. By analyzing this, we can determine the particular role that these select ion channels play in relation to the overall electrical conductance of the heart. This will allow us to determine if the neuronal circuit is disrupted in cardiac performance alterations, which can open doors for new perspectives in this work, such as manipulation. conclusion that among the ion channels selected, there is a correlation that allows the genes for ion channels in the cardiac ICN to play a role in the electrical conductance in the heart of a rat. This analysis was also observed in other rat and pig hearts which leads to the belief that there is indeed a key role of ion channels in the cardiac neuronal circuit which regulates electrical conductivity in the heart.

\section{Acknowledgements}

Enormous gratitude to Dr. Rajanikanth Vadigepalli, Alison Moss, Shaina Robbins, and Sean Nieves for guiding me through this project.

\section{References}

1. CV Physiology | Sinoatrial Node Action Potentials. (n.d.). https://www.cvphysiology.com/Arrhythmias/ A004 (accessed Apr 1, 2020)

2. Armour, A. (2007, February). The Little Brain of the Heart. Retrieved from https://fohs.bgu.ac.il/develop/DB2

/Heart memory/Armour, 2007.pdf (accessed Apr 1, 2020).

3. Shen, M. J.; Fishbein, M. C.; Chen, S. L.; Chen, P.

Neural Activity and Atrial Tachyarrhythmias. https://linkinghub.elsevier.com/retrieve/ pii/B9780323447331000407 (accessed Apr 1, 2020).

4. Roden, D. M., \& Kupershmidt, S. From genes to channels: normal mechanisms. OUP Academic. https://academic. oup.com/cardiovascres/article/42/2/318/

277458\#3418114 (accessed Apr 1, 2020).

5. Grant, A. O.; Grant, A. O.; Grant, A. O.; Grant, A. O. Cardiac Ion Channels. https://www.ahajournals.org/ doi/full/10.1161/circep.108.789081 (accessed Apr 1, 2020).

6. Priest, B. T.; McDermott, J. S. Cardiac ion channels. https://www.ncbi.nlm.nih.gov/pmc/ articles/PMC4850049/ (accessed Apr 1, 2020).

7. Achanta, S., Gorky, J., Leung, C., Moss, A., Robbins, S., Eisenman, L., ... Schwaber, J. S. (2019). A Comprehensive Integrated Anatomical and Molecular Atlas of Rodent Intrinsic Cardiac Nervous System. doi: 10.1101/661033 www.biorxiv.org/ content/10.1101/661033v2.full (accessed Apr 1, 2020).

8. Bartos, D. C.; Grandi, E.; Ripplinger, C. M. Ion Channels in the Heart. https://www.ncbi.nlm.nih.gov/ pmc/articles/PMC4516287/ (accessed Apr 1, 2020)

9. Brack, K. E. The heart's 'little brain' controlling cardiac function in the rabbit. https://www.ncbi.nlm.nih.gov/ pmc/articles/PMC4409095/ (accessed Apr 1, 2020).

\section{- Author}

My name is Rohan Patil, and I am a junior in high school. I have a profound interest in neuroscience because of my fascination in the power and mystery of such a system. I plan to earn an MD and practice as a pediatric neurosurgeon.

\section{Conclusion}

In this study, several important markers that indicate that there is some sort of spatial distribution observed in specific genes for ion channels have been identified. This leads to the 